\title{
Comparisons of atmospheric data and reduction methods for the analysis of satellite gravimetry observations
}

\author{
E. Forootan, ${ }^{1}$ O. Didova, ${ }^{1,2}$ J. Kusche,${ }^{1}$ and A. Löcher ${ }^{1}$ \\ Received 21 May 2012; revised 23 January 2013; accepted 12 March 2013.
}

[1] The Gravity Recovery and Climate Experiment (GRACE) derived gravity solutions contain errors mostly due to instrument noise, anisotropic spatial sampling, and temporal aliasing. Improving the quality of satellite gravimetry observations, in terms of using more sensitive sensors and/or increasing the spatial isotropy, has been discussed in the context of the designed scenarios of future satellite gravimetry missions. Temporal aliasing caused by incomplete reducing of background models, however, is still a factor that affects the quality of the gravity field solutions. This paper specifically explores the possible physical, geometrical, and numerical modifications of the three-dimensional (3-D) integration approach to eliminate the high-frequency atmospheric effects from satellite gravimetry observations. The new modified 3-D approach is then applied to compute new sets of atmospheric dealiasing products, using atmospheric fields from the European Centre for Medium-Range Weather Forecasts (ECMWF) operational analysis model and ERA-Interim reanalysis. Impacts of modifications are compared to the prelaunch baseline and the current error-curve of GRACE as well as an error-curve of a Bender-type multiorbit satellite configuration. Specifically, we found that using latitude-dependent radius, latitude- and altitude-dependent gravity accelerations along with numerical modifications have a considerable impact on the 3-D integral. Comparing the new products to those of GRACE Atmosphere and Ocean Dealiasing level-1B shows a nonnegligible difference with respect to the prelaunch baseline of GRACE and a possible Bender-type mission up to harmonic degrees 13 and 50, respectively. A big difference is also found between the derived dealiasing products from ECMWF operational analysis and ERA-Interim indicating the importance of input parameters on the final atmospheric dealiasing products.

Citation: Forootan, E., O. Didova, J. Kusche, and A. Löcher (2013), Comparisons of atmospheric data and reduction methods for the analysis of satellite gravimetry observations, J. Geophys. Res. Solid Earth, 118, doi:10.1002/jgrb.50160.

\section{Introduction}

[2] The Gravity Recovery and Climate Experiment (GRACE) has provided valuable information about the global integral effects of mass redistributions within the Earth system [Kusche et al., 2012 and the references in it]. Yet, GRACE-derived gravity field solutions display errors due to instrument noise (K-band ranging system and GPS) [Sheard et al., 2012], anisotropic spatial sampling of the mission [Schrama et al., 2007; Sneeuw et al., 2004], and temporal aliasing caused by incomplete reduction of shortterm mass variations by models [Flechtner et al., 2010].

\footnotetext{
${ }^{1}$ Institute of Geodesy and Geoinformation, Bonn University, Bonn, North Rhine-Westphalia, Germany.

${ }^{2}$ Faculty Civil Engineering and Geosciences, Delft University of Technology, Delft, The Netherlands.

Corresponding author: E. Forootan, Institute of Geodesy and Geoinformation, Bonn University, Nussallee 17, D53115, Bonn, Germany. (forootan@geod.uni-bonn.de)

(C)2013. American Geophysical Union. All Rights Reserved. 2169-9313/13/10.1002/jgrb.50160
}

Increasing the spatial sampling isotropy has become a major issue in designing future missions dedicated to pursue the task of GRACE. The GRACE Follow-On (GRACE-FO) mission, scheduled for launch in 2017, still adopts the GRACE design of two satellites flying in one orbital plane. More sophisticated formation types are under discussion for a new generation of gravity missions beyond 2020. The options are investigated in a variety of studies, e.g., Wiese et al. [2009], Elsaka [2010], Anselmi [2010], and NG2 Team [2011]. These studies include single pair formations with a permanently changing link direction as well as parallel flights of two GRACE-type missions, e.g., combining one single pair in a polar orbit with another pair flying in a relatively lower inclination of $63^{\circ}$ as proposed by Bender et al. [2003]. Future gravity missions will thus have the potential to observe the temporal variations of the Earth's gravity field with a better accuracy, e.g., in Visser et al. [2010] and Elsaka et al. [2012].

[3] Isolating the long-term and seasonal hydrological signals is one of the major applications of time-variable satellite gravimetry products [Tapley et al., 2004]. Therefore, recovering monthly mean gravity field solutions, from the 
observations of satellite gravimetry missions, requires a careful reduction of the short-term (e.g., subdaily to monthly) variations of the atmosphere and the oceans due to their dominant effects [Han et al., 2004]. Within the processing of satellite gravimetry data, it is common to reduce the high-frequency nontidal oceanic and atmospheric mass redistributions from the level-1 measurements by using "background" models [Reigber et al., 2005; Flechtner, 2007; Mayer-Gürr et al., 2010a]. Otherwise, such highfrequency mass changes will be aliased into long wavelength signals leading to misinterpretation of hydrological signals [Velicogna et al., 2001].

[4] GeoForschungsZentrum (GFZ) Potsdam routinely produces nontidal high-frequency atmospheric and oceanic mass variation products called GRACE Atmosphere and Ocean Dealiasing level-1B (GRACE-AOD1B) products [Flechtner, 2007]. Karbon et al. [2011] showed that the current data-processing strategy of the GRACE-AOD1B [Flechtner, 2007] is sufficient for the current accuracy of GRACE monthly solutions. It should be mentioned here that, for the processing of the ITG-GRACE2010 monthly gravity solutions at Bonn University, the daily Kalman filterbased GRACE solutions [Kurtenbach et al., 2009] have been used to successfully improve the dealiasing procedure [Mayer-Gürr et al., 2010b].

[5] The dealiasing process, however, represents still a concern for the quality of the gravity field solutions. For instance, several studies show that the accuracy of GRACE is above the simulated prelaunch baseline [see, e.g., Meyer et al., 2010], for which the errors within the dealiasing procedure are believed to be one of the potential causes. Comparing surface pressure fields derived from the National Centers for Environmental Prediction (NCEP) and the European Center for Medium-Range Weather Forecast (ECMWF), Salstein et al. [2008] showed the high uncertainty of atmospheric products over most parts of Asia, Central Africa, high latitude oceans, and Antarctic. Through simulations, Thompson et al. [2004] showed that the impact of high-frequency atmospheric and oceanic errors can reach up to $2 \mathrm{~mm}$ in terms of geoid heights at a spatial resolution of $500 \mathrm{~km}$. Impacts of the atmospheric spatiotemporal variations and their errors on the observations of satellite gravimetry missions are discussed in Gruber et al. [2009], who stated that considering the uncertainties of computed atmospheric dealiasing products is crucial. Zenner et al. $[2010,2012]$ showed that including errors of the atmospheric and oceanic models in the procedure of computing dealiasing products has an impact that is strong enough to be sensed by the GRACE satellites.

[6] Based on the mentioned studies, therefore, improving the dealiasing products in order to reduce the temporal aliasing and obtain more accurate gravity fields is essential. The quest is even more critical for next-generation gravity missions which aim to determine the geoid with an accuracy of $1 \mathrm{~mm}$ [Anselmi, 2010; NG2 Team, 2011]. Nerem et al. [2006], Pierce et al. [2008], and Dehne et al. [2009] stated that, as a result of using more advanced laser-ranging devices in a GRACE-FO mission, the precision of the range rate measurements may be in the range of $\sim 0.6 \mathrm{nms}^{-1}$ instead of the current $\sim 0.2 \mu \mathrm{ms}^{-1}$ precision of the GRACE microwave system [see also Loomis et al., 2012]. Provided that all other short-term mass variations are perfectly known, a future four-satellite mission of the Bender-type configuration will even be sufficiently sensitive to detect $1 \mu \mathrm{m}$ degree variance error in a background model, up to degree 50 [NG2 Team, 2011]. In section 4, therefore, we will discuss the effects of geometrical, numerical, and physical approximations for computing the atmospheric dealiasing products which may pose a limitation for exploiting the full accuracy of the satellite gravimetry measuring systems.

[7] To improve dealiasing products, Flechtner et al. [2010] refer to experiments performed with the AOD RL04 baroclinic Ocean Model for Circulation and Tides (OMCT) model and three-hourly ECMWF forecasts. Although results appeared promising from theory, improvements were not clearly visible in the final gravity fields. Therefore, AOD RL05 is only based on a revised version of the OMCT model but still relies on six-hourly ECMWF atmospheric models (see, www.gfz-potsdam.de/aod1b).

[8] This paper, specifically, focuses on the atmospheric part of the dealiasing products, in which surface pressure, geopotential, temperature, and specific humidity fields from the ECMWF operational analysis (ECMWFop) are extracted and converted to potential coefficients. This conversion has been realized using a three-dimensional (3-D) integration approach including various approximations [Swenson and Wahr, 2002; Boy and Chao, 2005; Flechtner, 2007]. To investigate this procedure, first, the previous 3-D formulations of the atmospheric loading effects described in Boy and Chao [2005] and Flechtner [2007] were revisited (section 3.2). Then, possible modifications of the 3-D integration, with considering a more realistic physical and geometrical Earth's shape, were discussed (section 3.3). Numerical aspects of the computations were also investigated and improved (section 3.4). All physical and geometrical assumptions within the modified 3-D model were compared to the prelaunch baseline and the current errorcurve of GRACE monthly fields (section 4). In addition, a predicted error-curve of a Bender-type satellite configuration was used to assess the effect of atmospheric dealiasing products on the scenario of future gravimetry missions [NG2 Team, 2011].

[9] The main changes within the new 3-D integration method, called "ITG-3D" in this paper, over the common approach used for computing the atmospheric part of GRACE-AOD1B are threefold: (1) geometrical modification including ellipsoidal radius $r_{e}(\theta)$ instead of a constant radius, incorporating geoid heights from the ITGGRACE2010s static solution instead of using the surface geopotential from ECMWF, as well as using a more accurate transformation for computing radial coordinates [Office of the Federal Coordinator for Meteorological Services and Supporting Research (OFCM), 1997]; (2) physical modification including the more accurate latitudeand altitude-dependent gravity acceleration formula by Heiskanen and Moritz [1967] within the vertical integration instead of a simple linear approximation of the latitudedependent gravity acceleration; and finally, (3) numerical improvements, i.e., considering subintervals between each model level for better performing the vertical integration, as well as using the Gauss-Legendre Quadrature (GLQ) method [Krylov, 1962] for improving the computation of the desired atmospheric dealiasing spherical harmonics by horizontal integration. 
[10] The modified 3-D integration approach was then used to compute new sets of atmospheric dealiasing products based on ECMWFop and ERA-Interim, covering the period 2001 to 2009 (sections 4.2 and 4.3). The impacts of input atmospheric parameters on the new products were numerically assessed by comparing the atmospheric dealiasing products derived from ECMWFop to those of ERA-Interim (section 4.3).

[11] The remaining part of the manuscript is organized as follows: Atmospheric data derived from the ECMWFop along with ERA-Interim reanalysis data are described in section 2. In section 3, first, the previous formulations of the atmospheric loading effects described in Boy and Chao [2005] and Flechtner [2007] based on 3-D approaches are revisited (section 3.2), and their possible modifications are discussed (sections 3.3 and 3.4). Section 4 is devoted to the numerical results of the study, including the impact of assumptions on radial coordinates and gravity acceleration, the influence of each physical and geometrical assumptions on the modified ITG-3-D method as well as the impacts of atmospheric parameters on the new computed atmospheric dealiasing products. Section 5 discusses our findings and concludes the study.

\section{Data}

\subsection{ECMWFop}

[12] ECMWFop is one of the premiere models for medium-range and seasonal-forecasting purposes [Stockdale et al., 1998]. This study used the atmospheric fields of ECMWFop, from 2001 to 2009, including pressure $P_{s}$ and geopotential $\Phi_{s}$ over the Earth's surface as well as temperature $T$ and specific humidity $S$ defined at model levels $k$. Since 1 February 2006, the number of levels in the model has been increased from $k=0: 60$ to $k=$ $0: 91$. This change improved the vertical resolution of the model to better account for the main atmospheric mass changes within the lower and midtroposphere (where the pressure layers follow the Earth's surface). With 91 model levels, the top model coverage of ECMWFop was extended from $0.1 \mathrm{hPa}(\sim 65 \mathrm{~km})$ to $0.01 \mathrm{hPa}(\sim 80 \mathrm{~km})$. Atmospheric parameters among others are available on a reduced Gaussian grid (N160 grids with spatial resolution of about $0.5^{\circ}$ ) with temporal resolution of every $6 \mathrm{~h}$ (at 00:00 h, 06:00 h, 12:00 h, and 18:00 h). Details about the reduced Gaussian grids can be found from ECMWF website (http://www.ecmwf.int/products/data/technical/gaussian/). ECMWFop products were downloaded from the Information System and Data Center (ISDC), GeoForschungsZentrum (GFZ), Potsdam (http://isdc.gfz-potsdam.de/ index.php).

\subsection{ERA-Interim}

[13] ERA-Interim is the latest global atmospheric reanalysis data set, produced by ECMWF, covering the period 1989 up to now. The quality of ERA-Interim has been improved as a result of better modeling, input data, and assimilation methods. Lorenz and Kunstmann [2012] recently pointed out that ERA-Interim, when compared to other reanalysis products, e.g., Modern Era Retrospective-Analysis for Research and Applications (from the National Aeronautics and Space Administration) and Climate Forecast System Reanalysis (from NCEP), shows more reasonable closure of the terrestrial and atmospheric water balance as well as higher agreement with in situ data sets. ERA-Interim, therefore, provides a global model of atmospheric changes that encompasses many essential climate variables in a physically consistent framework, with only a short time delay compared to operational models [Dee et al., 2011]. This study used the ERA-Interim data obtained from the official website of ECMWF (http://www.ecmwf.int/research/era/do/get/ era-interim). These products are the reanalyzed version of the ECMWF data assimilation and forecasting products, while the vertical levels are 37 pressure levels [Berrisford et al., 2009]. The required parameters of ERA-Interim are available on N128 reduced Gaussian grids (about $0.7^{\circ}$ spatial resolution) with temporal resolution of every $6 \mathrm{~h}$ (at 00:00 h, 06:00 h, 12:00 h, and 18:00 h).

\section{Methodology}

[14] Atmospheric dealiasing products are the sets of gravity spherical harmonic coefficients $\Delta C_{n m}(t)$ and $\Delta S_{n m}(t)$, computed up to degree $n$ and order $m$ (e.g., in GRACEAOD1B, $m=n=100)$, which reflect the atmospheric spatiotemporal mass variations for a certain time $t$ [Boy and Chao, 2005; Flechtner, 2007]

$$
\left.\begin{array}{c}
\Delta C_{n m}(t) \\
\Delta S_{n m}(t)
\end{array}\right\}=\frac{\left(1+k_{n}^{\prime}\right) a^{2}}{(2 n+1) M} \iint_{\sigma} \Delta I_{n}(\theta, \lambda, t) P_{n m}(\cos \theta)\left\{\begin{array}{c}
\cos m \lambda \\
\sin m \lambda
\end{array}\right\} \mathrm{d} \sigma .
$$

In equation (1), $k_{n}^{\prime}$ are the load Love numbers [Dong et al., 1996; Farrel, 1972], $a$ is the semi-major axis of the reference ellipsoid [e.g., the Geodetic Reference System 1980 (GRS80)], $M$ is the mass of the Earth, $\theta$ and $\lambda$ are the colatitude and longitude, $t$ is the time, $d \sigma$ is the surface element, and $P_{n m}$ are the fully normalized associated Legendre polynomials. The factor $1+k_{n}^{\prime}$ takes into account the direct effect of gravitational attraction by air mass and the indirect elastic loading effect [Boy and Chao, 2005]. The vertical integral $\Delta I_{n}(\theta, \lambda, t)$ in equation (1) reflects atmospheric mass variations and is derived from

$$
\begin{aligned}
\Delta I_{n}(\theta, \lambda, t) & =\left(\int_{a}^{\infty}\left(\frac{r}{a}\right)^{n+2} \rho(\theta, \lambda, r, t) \mathrm{d} r\right)-\bar{I}_{n}(\theta, \lambda) \\
& =I_{n}(\theta, \lambda, t)-\bar{I}_{n}(\theta, \lambda),
\end{aligned}
$$

where $r$ is the distance to the Earth's center of mass, $\rho(\theta, \lambda, r, t)$ models the atmospheric density distributions. $\bar{I}_{n}(\theta, \lambda)$ is a degree-dependent mean field that should be subtracted from the integral in order to remove the long wavelength atmospheric variations. In practice, the term $\bar{I}_{n}$ is a mean field from vertical integration that is computed by the temporal averaging of equation (2), without considering the $\bar{I}_{n}$ term, over a certain period. Consequently, geoid height variations $(\Delta N)$ caused by atmospheric mass variations can be computed following the approach of Wahr et al. [1998].

\subsection{Relationship Between Density and Atmospheric Parameters}

[15] In practice, a direct measurement of the density term, $\rho(\theta, \lambda, r, t)$ in equation (2), is not available. Therefore, for computing $I_{n}$ [equation (2)], it is common to use the conventional relationship between the multilevel outputs of operational atmospheric models and density changes 
Table 1. Formulations of Inner Integrals $\left(I_{n}(\theta, \lambda)\right)$ Used in 3-D Methods

\begin{tabular}{ll}
\hline Spherical Earth [equation (8)] & $\int_{0}^{P_{s}}\left(\frac{a}{a-\Phi_{k+1 / 2}^{g}}+\frac{\xi(\theta, \lambda)}{a}\right)^{n+4} \frac{\mathrm{d} P(\theta, \lambda)}{g}$ \\
AOD1B [equation (13)] & $\int_{0}^{P_{s}}\left(\frac{a}{a-\Phi_{k+1 / 2}^{g} \frac{g}{g(\theta)}}+\frac{\xi(\theta, \lambda)}{a}\right)^{n+4} \frac{\mathrm{d} P(\theta, \lambda)}{g}$ \\
ITG-inner integral [equation (15)] & $\int_{0}^{P_{s}}\left(\frac{r_{e}(\theta)+z_{k+1 / 2}+\xi(\theta, \lambda)}{a}\right)^{n+2} \frac{\mathrm{d} P(\theta, \lambda)}{g\left(\theta, z_{k+1 / 2}\right)}$ \\
\hline
\end{tabular}

[White, 2001]. In fact, most of the numerical atmospheric models with spatial resolution of larger than $\sim 20 \mathrm{~km}$ [Haltiner and Williams, 1979] use the hydrostatic equation (i.e., air pressure at any height in the atmosphere is caused by the weight of all of the air mass above that height) to approximate global atmospheric flow [Gill, 1999]. Therefore, the relationship between pressure and height is derived from

$$
\rho d z=\frac{-d P}{g},
$$

with $g$ being the mean Earth surface gravity acceleration, $d P$ is the vertical pressure changes, and $z$ is the height from the surface of the Earth.

[16] Replacing $I_{n}$ in equation (2) with the right-hand side of equation (3), therefore, can be done with various assumptions such as considering only a thin layer pressure (known as 2-D methods) or considering the vertical structure of atmosphere (known as 3-D methods) [cf. Boy and Chao, 2005].

\subsection{Revisiting Previous Three-Dimensional (3-D) Methods}

[17] Several studies have shown that for removing atmospheric mass variation from satellite gravimetry observations, the 3-D structure of the atmosphere cannot be neglected [e.g., Boy and Chao, 2005; Flechtner, 2007; Gruber et al., 2009]. Assuming that there is no vertical pressure gradient between model levels, $I_{n}$ on the spherical Earth is derived from the downward integration of the gravitational force acting on a column of atmospheric air

$$
I_{n}(\theta, \lambda)=\int_{0}^{P_{s}}\left(\frac{r}{a}\right)^{n+2} \frac{\mathrm{d} P(\theta, \lambda)}{g(z)} .
$$

In equation (4), $g(z)$ has been implemented as $g(z)=g\left(\frac{a}{r}\right)^{2}$, with $g$ being the constant gravity acceleration at the equator and $r=a+z$ [Swenson and Wahr, 2002]. Note that the distance of a point to the Earth's center of mass $(r)$ on a more realistic Earth is approximately

$$
r=r_{e}(\theta)+\xi(\theta, \lambda)+z(\theta, \lambda)
$$

with ellipsoidal radius $r_{e}(\theta)$, geoid height $\xi(\theta, \lambda)$, and orthometric height of the point above the mean geoid $z(\theta, \lambda)$ [Peters, 2007].

[18] Since in atmospheric models, the vertical coordinate is the geopotential height instead of the geometrical height [White, 2001], for computing equation (4), Flechtner [2007] formulated the relationship between geopotential height (denoted here by $\Phi^{\mathrm{g}}$ ) and altitude $z$ as

$$
\Phi^{g}=\frac{1}{g} \int_{0}^{z} g(z) \mathrm{d} z=\frac{a z}{a+z}
$$

and therefore,

$$
z=\frac{\Phi^{g}}{1-\frac{\Phi^{g}}{a}}
$$

[19] In equations (6) and (7), the spatial variability of $g$ is neglected. Thus, $\Phi^{\mathrm{g}}$ is only altitude-dependent.

[20] Having computed $z$ from equation (7) and replacing $r_{e}$ in equation (5) by the major semi-axis of the reference ellipsoid yields $r(\theta, \lambda)=\frac{a}{1-\frac{\Phi g}{a}}+\xi(\theta, \lambda)$. Thus, $I_{n}$ from equation (4) becomes

$$
I_{n}(\theta, \lambda)=\int_{0}^{P_{s}}\left(\frac{a}{a-\Phi_{k+1 / 2}^{g}}+\frac{\xi(\theta, \lambda)}{a}\right)^{n+4} \frac{\mathrm{d} P(\theta, \lambda)}{g},
$$

where $k$ is the model level. Since in the last equation, the distance of the points are referred to a spherical reference surface $\left(r_{e}=a\right)$, and $g$ is assumed to be constant, equation (8) is called "3D-Spherical-Earth" in Table 1. To compute pressure changes $d P$ in equation (8), air pressure is derived at model boundaries, using the "model constants" $a_{k+1 / 2}$ and $b_{k+1 / 2}$ (provided by atmospheric models) as follows:

$$
P_{k+1 / 2}=a_{k+1 / 2}+b_{k+1 / 2} P_{s},
$$

with $P_{s}$ being the surface pressure [White, 2001]. The model constants reflect the vertical coordinate of the atmospheric model in which the model equations are discretized. $\Phi_{k+1 / 2}^{g}$ in equation (8) is derived as

$$
\Phi_{k+1 / 2}^{g}=\Phi_{s}^{g}+\frac{1}{g} \sum_{j=k+1}^{\mathrm{NLEV}} R_{\mathrm{dry}}\left(T_{v}\right)_{j} \ln \frac{P_{j+1 / 2}}{P_{j-1 / 2}}
$$

[Simmons and Burridge, 1981].

[21] $T_{v}$ is the virtual temperature derived from

$$
T_{v}=(1+0.608 S) T,
$$

with $S$ and $T$ being respectively the specific humidity and temperature derived from atmospheric models [White, 2001; Flechtner, 2007].

[22] According to the conventions of the World Meteorological Organization [WMO, 2006], the theoretical value of the gravity acceleration that is used in equation (6) should be derived from local acceleration of gravity at a certain point with consideration of a suitable Bouguer correction [Heiskanen and Moritz, 1967]. Alternatively, this value can be replaced from a geodetic model, e.g., GRS80 [WMO, 2006]. Fagiolini et al. [2007] suggested to use a linear approximation of the latitude-dependent gravity from Heiskanen and Moritz [1967], i.e.,

$$
g(\theta)=\gamma_{e}+\left(\gamma_{p}-\gamma_{e}\right) \cos ^{2} \theta,
$$

with $\gamma_{e}$ and $\gamma_{p}$ being the normal gravity accelerations on the equator and poles, respectively. Inserting $g(\theta)$ in 


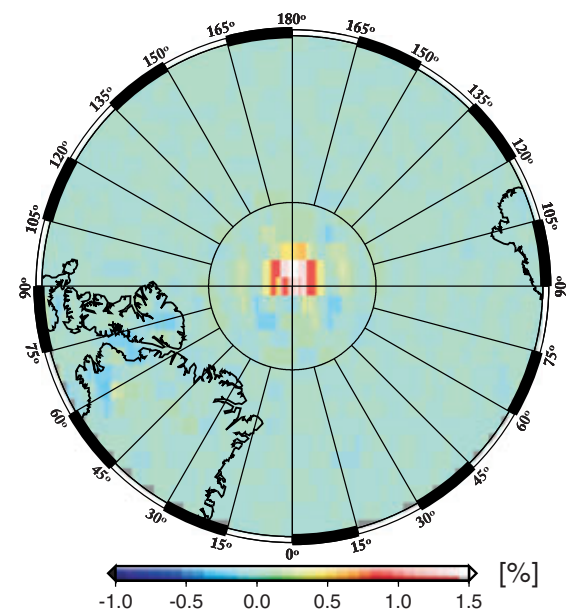

(a)

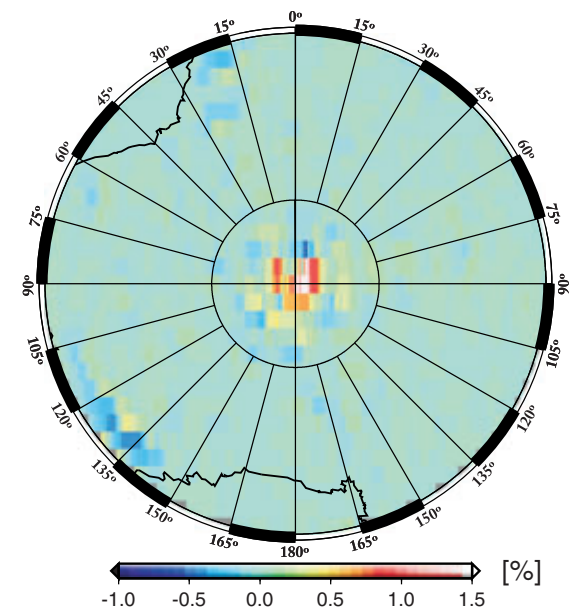

(b)

Figure 1. The differences between the error percentage of an ordinary 2-D integration and the error percentage of GLQ for synthesizing a pressure field on 1 January 2001 at 00:00 h. Figure 1a shows the errors toward the North Pole $\left(75^{\circ} \mathrm{N}<\right.$ latitude $\left.<90^{\circ} \mathrm{N}\right)$, and Figure $1 \mathrm{~b}$ shows the errors toward the South Pole $\left(75^{\circ} \mathrm{S}<\right.$ latitude $\left.<90^{\circ} \mathrm{S}\right)$. In both graphs, red color shows those regions in which the ordinary integration for computing spherical harmonics exhibits larger error percentages, while the blue color shows the opposite.

equation (8), the inner integral of the GRACE-AOD1B products was modified (F. Flechtner (GFZ, Potsdam), 2012 pers. communication) as

$$
I_{n}(\theta, \lambda)=\int_{0}^{P_{s}}\left(\frac{a}{a-\Phi_{k+1 / 2}^{g} \frac{g}{g(\theta)}}+\frac{\xi(\theta, \lambda)}{a}\right)^{n+4} \frac{\mathrm{d} P(\theta, \lambda)}{g} .
$$

Note that $\xi(\theta, \lambda)$ in equations (8) and (13) has been derived from surface geopotential data, using atmospheric models, $\xi(\theta, \lambda)=\frac{\Phi_{s}}{g}$.

[23] Equation (13) ("3D-AOD1B" in Table 1) is mathematically equivalent to the one Boy and Chao [2005] suggested to estimate $I_{n}$

$$
I_{n}(\theta, \lambda)=\int_{0}^{P_{s}}\left(\frac{a+z_{k+1 / 2}+\xi(\theta, \lambda)}{a}\right)^{n+2} \frac{\mathrm{d} P(\theta, \lambda)}{g\left(\theta, z_{k+1 / 2}\right)} .
$$

\subsection{Modifying the Formulation of 3-D Methods}

[24] This study suggests to modify $I_{n}$ in equation (14) with considering a more realistic geometrical and physical shape of the Earth, i.e., (1) using equation (5) with $r_{e}(\theta)$ from GRS80 and geoid height $\xi$ from ITG-GRACE2010s, and (2) using a better approximation of the latitude- and altitudedependent gravity acceleration in equation (14). Therefore, equation (14) is modified as

$$
I_{n}(\theta, \lambda)=\int_{0}^{P_{s}}\left(\frac{r_{e}(\theta)+z_{k+1 / 2}+\xi(\theta, \lambda)}{a}\right)^{n+2} \frac{\mathrm{d} P(\theta, \lambda)}{g\left(\theta, z_{k+1 / 2}\right)},
$$

which is referred as "3-D-ITG-inner integral" in Table 1. The impacts of the suggested improvements in equation (15) are discussed in section 4 .

[25] Following Boy and Chao [2005], we compute equation (15) based on the (vertical) sigma coordinates while considering the following items:

[26] 1. Similar to 3-D-AOD1B, pressure values at all model boundaries were computed from equation (9).
[27] 2. Virtual temperatures at each model levels were computed from equation (11).

[28] 3. Combining the hydrostatic equation and the gas law [White, 2001], geopotential heights for all model levels were derived from equation (10) with considering a rescaled geopotential $\left(\Phi_{s}^{g}=\Phi_{s} / g_{45}\right)$, according to $W M O$ [2006].

[29] 4. Transforming the geopotential heights into geometric heights was done according to the conventions of OFCM [1997, Appendix D],

$$
z_{k+1 / 2}=\frac{r_{e}(\theta) \Phi_{k+1 / 2}^{g}}{\frac{g(\theta) r_{e}(\theta)}{g}-\Phi_{k+1 / 2}^{g}},
$$

with ellipsoidal radius $r_{e}(\theta)$. Unlike 3-D-AOD1B, the latitude-dependent gravity $g(\theta)$ in equation (16) was derived using the second-order expansion of $g(\theta)$ [Heiskanen and Moritz, 1967], with incorporating flattening $(f)$, the ratio of the centrifugal acceleration $(m)$ and the normal gravity at the equator $\left(\gamma_{e}\right)$ from GRS80,

$$
g(\theta)=\gamma_{e}\left(1+f_{2} \cos ^{2} \theta+f_{4} \cos ^{4} \theta\right),
$$

with $f_{2}=-f+\frac{5}{2} m+\frac{1}{2} f^{2}-\frac{26}{7} f m+\frac{15}{4} m^{2}, f_{4}=-\frac{1}{2} f^{2}+\frac{5}{2} f m$ [Heiskanen and Moritz, 1967, p. 79]. The impact of $g(\theta)$, derived from equation (17), on atmospheric dealiasing products is discussed in section 4.1 .

[30] 5. The latitude- and altitude-dependent gravity acceleration in equation (15) was derived from

$$
g\left(\theta, z_{k+1 / 2}\right)=g(\theta)\left[1-\frac{2}{a}\left(1+f+m-2 f \cos ^{2}(\theta)\right) z_{k+1 / 2}+\frac{3}{a^{2}} z_{k+1 / 2}^{2}\right] .
$$

\subsection{Improving the Numerical Integration}

\subsubsection{Resolving Spherical Harmonics From} Gridded Data

[31] Once the inner integral $I_{n}$ was computed from one of the methods in Table 1, equation (2) will 


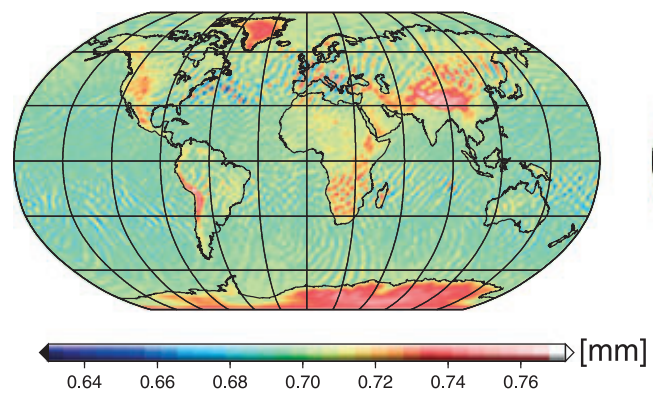

(a)

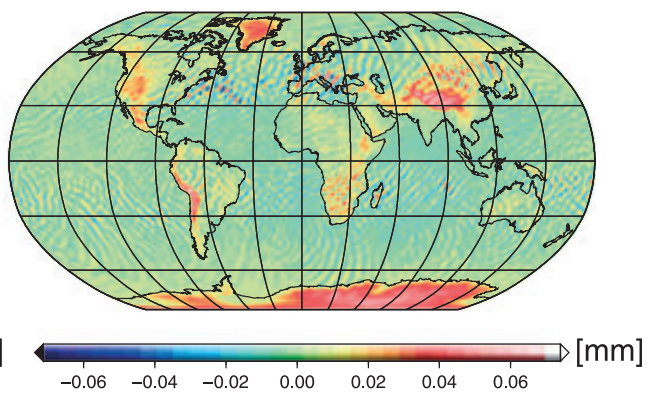

(b)

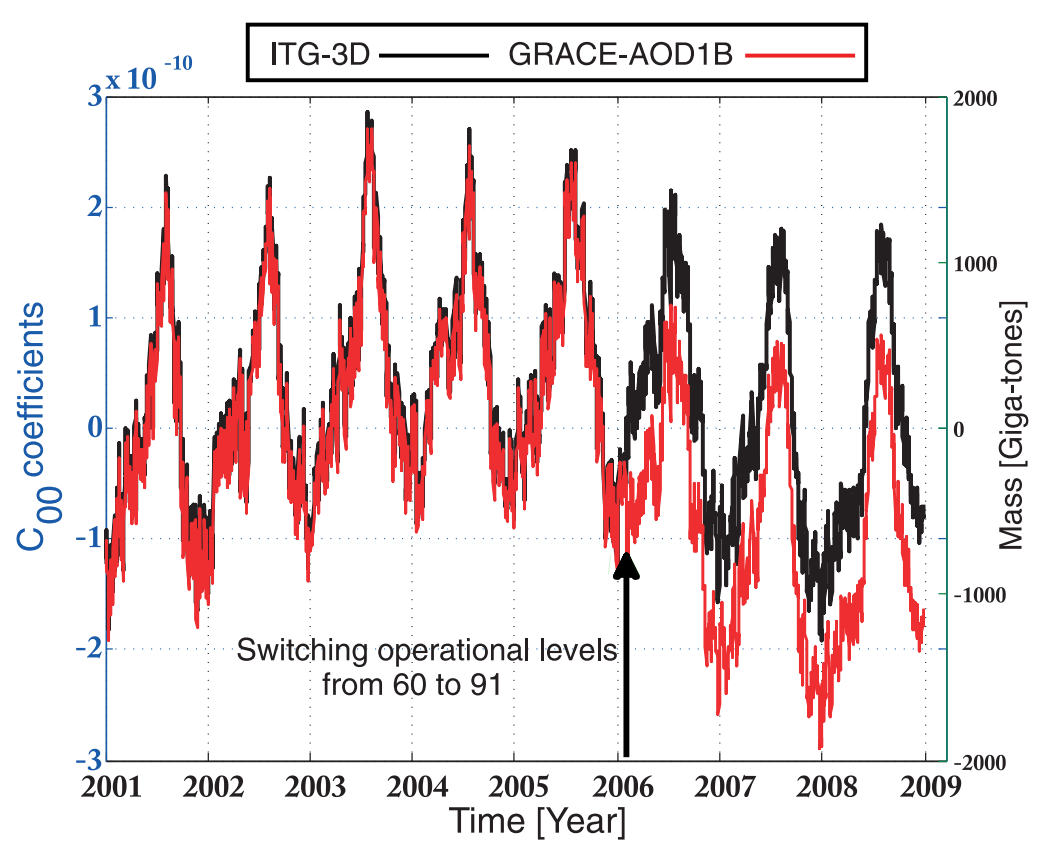

(c)

Figure 2. Overview of the difference between considering and not considering the vertical refinements on computing $I_{n}$ [equation (15)]. (a) The differences in terms of standard deviation of geoid height including the degree one and zero coefficients, based on the ECMWFop data on 1 January 2001 at 00:00 h; (b) the same impact as in Figure 2a without including the degree one and zero coefficients; and (c) time series of $C_{00}$ from ITG-3-D and from GRACE-AOD1B.

be evaluated. For deriving the desired spherical harmonics from equation (1), therefore, one will have to apply a 2-D integration on gridded spatial domains. For computing spherical harmonics of GRACE-AOD1B products, Flechtner [2007] used a bilinear interpolation (http://www.ecmwf.int/publications/manuals/libraries/ interpolation/index.html) to transform the atmospheric parameters of ECMWFop from their original reduced Gaussian grids to equiangular grids with $0.5^{\circ}$ resolution. Then, the regular grids were smoothed by computing block mean values. The desired spherical harmonics, finally, were derived using an ordinary 2-D integration in equation (1) [Flechtner, 2007]. Note that performing block mean values before implementing the 2-D integration considerably improves the numerical derivations of spherical harmonics [Fagiolini et al., 2007]. Similar arguments, for improving the performance of 2-D integration on nonsmooth data sets, can be found in, e.g., Wang et al. [2006].
[32] Using a synthetic example, however, one can see that implementing an ordinary integration method on regular grids provides less accurate zonal coefficients, i.e., the maximum computation errors are distributed toward the poles [Colombo, 1981; Sneeuw, 1994]. To reduce such behavior, this study used the Gauss-Legendre Quadrature (GLQ) method as it is described in Krylov [1962] to derive spherical harmonics [from equation (1)]. A quadrature rule is an approximation of the 2-D integral as a weighted sum of the specified points within the domain of integration. Keeping in mind that in an ordinary integration, the numerical summation is equally weighted, Colombo [1981] shows that introducing weights within the GLQ approach, improves the performance of 2-D integration for computing spherical harmonics from sampled grid points (for details, see, e.g., Eicker [2008], pp. 80-82).

[33] To illustrate what we gain with implementing GLQ instead of an ordinary 2-D integration for computing the 
final products, we synthesized a surface pressure field on 1 January 2001 at 00:00 h up to degree and order 100 using an ordinary 2-D integration and GLQ. Before implementing the integrations, for both approaches, the pressure field was transformed to an $0.5^{\circ}$ equiangular grid and smoothed by computing block mean values. Our results showed that for $70 \%$ of the grid points, the synthesized values from GLQ exhibit fewer errors than the results of an ordinary 2-D integration. These differences are bigger in the high-latitude regions $\left(75^{\circ} \mathrm{N}<\right.$ latitude $<90^{\circ} \mathrm{N}$ and $75^{\circ} \mathrm{S}<$ latitude $<$ $90^{\circ} \mathrm{S}$ ), where Figure 1 shows the differences between the error percentage of the ordinary integration and the error percentage of GLQ. The magnitudes of the differences in Figure 1 reach up to $2 \%$ error percentage around the polar regions, which is equivalent to $\sim 0.5 \mathrm{hPa}$.

\subsubsection{Inner Integral}

[34] Atmospheric parameters such as specific humidity and temperature do not change linearly from the Earth's surface to the highest model level [see, e.g., White, 2001; $W M O, 2006]$. Therefore, the terms within the inner integral $I_{n}$ [e.g., in equation (15)] do not vary linearly with respect to the pressure changes $d P$. To improve the numerical performance of the inner integral, we refined the vertical resolution by considering $i=5$ subintervals between each model level $\left(d P=\sum_{i=1}^{5} \delta p_{i}\right)$. Then, the values of $I_{n}$ were linearly interpolated to the new subintervals, and it was numerically solved. $i=5$ was found experimentally as an optimal number of subsections which compromised the computation time and the improvement of the results. Note that by introducing subintervals, we aim at improving the accuracy of the numerical integration of the vertical integral. This does not add actual information to the model itself nor is it meant to smooth the profiles of the model.

[35] Figures $2 \mathrm{a}$ and $2 \mathrm{~b}$ show the difference between computing equation (15) with and without vertical refinements, in terms of geoid height using the ECMWFop data on 1 January 2001 at 00:00 h. The spatial difference shows a mean difference, which with considering the degree one and zero coefficients reaches up to $0.7 \mathrm{~mm}$ and without considering them reaches up to $0.07 \mathrm{~mm}$, in terms of geoid heights. In the following, the approach that is used for computing the desired atmospheric dealiasing spherical harmonics [equation (1)] with considering $I_{n}$ from equation (15) (3-D-ITG-inner integral in Table 1), while the mentioned vertical refinement and GLQ are applied, is called ITG-3-D approach.

[36] To study the temporal effect of the vertical refinement, we used the ECMWFop data for the period of 2001 to 2009. Then, atmospheric dealiasing coefficients were computed using the ITG-3-D approach. Since $C_{00}$ is linked to the total atmospheric mass change $\left(M=\frac{a^{2} C_{00} g}{G}\right.$, with $a$ being the equatorial radius of the Earth and $G$ is the gravitational constant), the time series of $C_{00}$ computed from ITG-3-D along with $C_{00}$ from GRACE-AOD1B are shown in Figure 2c. The results indicate that our estimation of the atmospheric $C_{00}$ is less sensitive than GRACE-AOD1B products to the leap caused by switching ECMWF's levels from 60 to 91 on 1 February 2006. Note that the same argument is also true for the other computed coefficients. However, the other coefficients, themselves, are much less sensitive to the switching of the number of model levels. The derived results
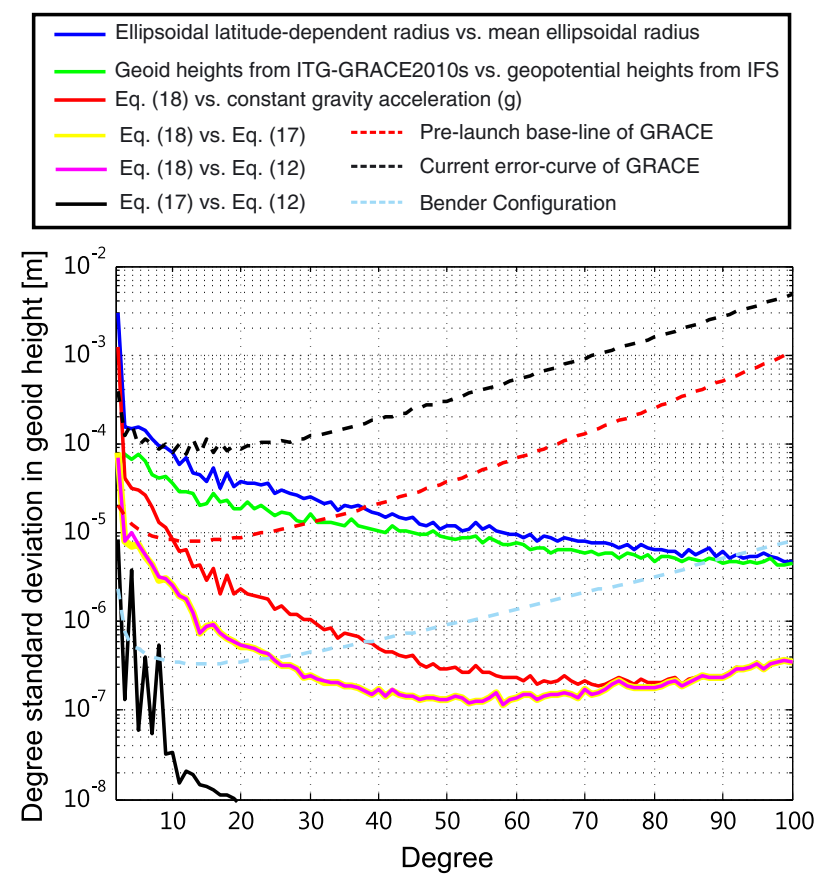

Figure 3. Impact of different geometrical and physical assumptions on atmospheric dealiasing products, based on the ECMWFop data on 1 January 2001 at 00:00 h, in terms of degree standard deviations of geoid height. Dotted lines show the sensitivity of gravity missions. Solid blue line denotes the difference between using a latitudedependent radius $r_{e}(\theta)$ instead of the semi-major axis of GRS80 (a) within equation (15). This impact was caused by the major geometrical difference between our suggested equations (15) and (14). Solid green line denotes the difference between using geoid heights from ITG-GRACE2010s to compute the distance of atmospheric computation point to the Earth's center of mass instead of converting geopotential heights from the operational model. Solid red line denotes the difference between using a latitude- and altitudedependent [equation (18)] and a constant gravity acceleration. This impact is counted as the physical modification. Solid magenta and solid yellow lines denote the impact of using $g(\theta, z)$ instead of latitude-dependent gravity acceleration $g(\theta)$ derived from equations (12) and (17), respectively. Solid black line denotes the difference between using $g(\theta)$ from equations (17) and (12).

in Figure 2c also convinced us that the vertical refinement was a proper choice because the temporal evolution of the atmospheric mass (in ITG-3-D) is less affected by the bias between the model with 60 and 91 levels. We feel this is relevant since it proves that switching the model levels from 60 to 91 mainly refines the vertical resolution of the model and probably does not represent a significant change of the global atmospheric mass distribution itself.

\section{Numerical Results}

\subsection{Impact of Geometrical and Physical Assumptions on 3-D Integration}

[37] As discussed in section 3, the geometric distance of the atmospheric computation points to the Earth's 


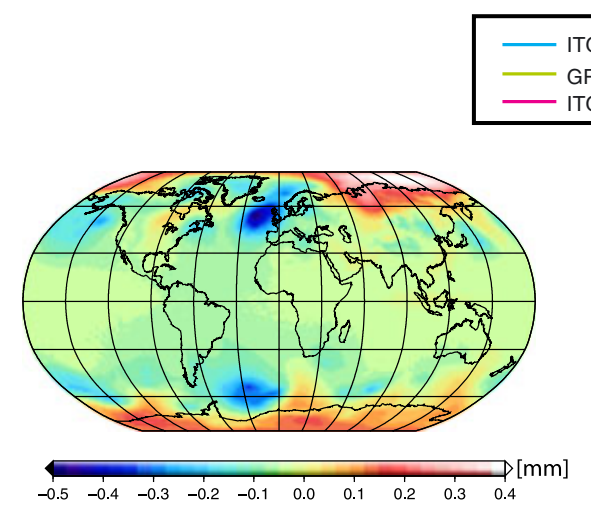

(a)
- - - Pre-launch baseline of GRACE - - - Current error-curve of GRACE Bender Configuration

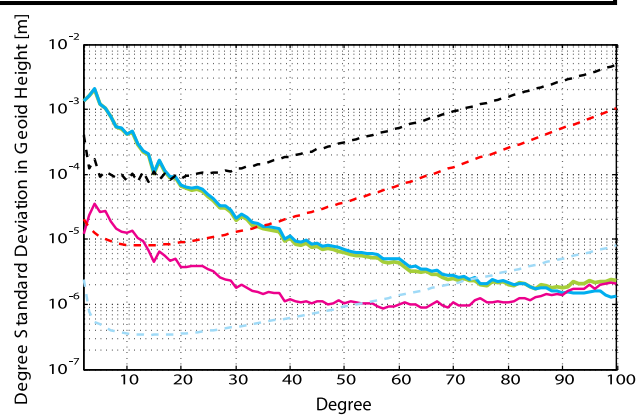

(b)

Figure 4. Comparison between ITG-3-D method and GRACE-AOD1B, based on the ECMWFop data on 1 January 2001 at 00:00 h. (a) Spatial pattern, in terms of geoid heights and (b) spectral representation of Figure $4 \mathrm{a}$, in terms of degree standard deviations of geoid height. Dotted lines show the sensitivity of gravity missions. Solid blue line denotes the dealiasing product computed using ITG-3-D. Solid green line denotes the atmospheric part of GRACE-AOD1B. Solid magenta line denotes the difference between ITG-3-D [inserting equation (15), with considering the discussed numerical improvements from section 3.4, in equation (1)] and GRACE-AOD1B [inserting equation (13), without further numerical modifications, in equation (1)]. The difference is above the prelaunch baseline of GRACE and the instrumental error-curve of a Bender mission up to degrees 13 and 50, respectively.

center of mass and the assumptions of gravity acceleration of the Earth are two factors within the introduced 3-D modeling approaches in Table 1. To compare these impacts with the sensitivity of satellite gravimetry missions, we computed $I_{n}$ [equation (15)] with respect to the different assumptions on the radius of the Earth, geoid heights, and gravity acceleration. Figure 3 shows the results, in terms of standard deviations for geoid heights, using the ECMWFop data on 1 January 2001 at 00:00 h.

[38] Note that, for all the comparisons presented in this study, the pre-launch baseline of GRACE was derived from the results of $\mathrm{Kim}$ [2000], while its actual monthly errorcurve was taken from ITG-GRACE2010 unconstrained monthly solutions [Mayer-Gürr, 2006 and Mayer-Gürr et al., 2010c]. The error-curve of the Bender configuration was computed in a full-scale simulation taking into account all critical instrumental errors, but neglecting any mismodeling of the short-term mass variations (dealiasing errors) [NG2 Team, 2011]. Even for a mission with launch date after 2020, this assumption is optimistic, but it relates the errorcurve directly to the GRACE baseline which was computed in the same way [cf. Kim, 2000].

[39] From our results, the largest impact is related to the difference between using a latitude-dependent radius $r_{e}(\theta)$ instead of the semi-major axis of $\operatorname{GRS} 80(a)$ within equation (15). This impact was caused by the major geometrical difference between equation (14) and our suggested equation (15), which is found above the current error-curve of GRACE up to degree 9. The prelaunch baseline errors of GRACE and the instrumental error-curve of a possible Bender-type configuration respectively are below the mentioned geometrical effect up to degrees 37 and 90. This result shall prove the importance of using the latitudedependent radius in computing atmospheric dealiasing products for reducing the current GRACE observation. The large magnitude of this impact is due to the difference between the ellipsoidal and spherical radius which reaches up to maximum $22 \mathrm{~km}$ (Figure 3).

[40] The impact of using geoid heights from ITGGRACE2010s to compute the distance of the atmospheric computation point to the Earth's center of mass in equation (15), instead of converting geopotential heights from ECMWFop was found below the current error-curve of GRACE. It was, however, above the prelaunch baseline of GRACE and the instrumental error-curve of a Bender mission up to degrees 31 and 89 , respectively. These results emphasize the importance of geometrical assumptions on the final dealiasing products.

[41] The difference between using a latitude- and altitudedependent and a constant gravity acceleration $[g(\theta, z)$ and $g]$ in $I_{n}$ was found above the current error-curve of GRACE, its prelaunch baseline and the error-curve of Bender up to degrees 4,10 , and 39 , respectively. We also computed the differences between latitude- and altitude-dependent gravity acceleration $[g(\theta, z)]$ and two different formulations of latitude-dependent gravity acceleration [equations (12) and (17)]. The differences were above the current error-curve and the prelaunch baseline of the GRACE mission up to degree 3 and above of a Bender-type mission up to degree 25 . The difference between using $g(\theta)$ from equation (17) and the linear approximation of $g(\theta)$ [equation (12)] itself has a nonnegligible impact on the low-degree coefficients of a Bender mission, especially for the zonal coefficients up to degree 8 . Since the largest magnitude of the difference was detected for the $C_{20}$-coefficient, considering this impact for reducing GRACE observations might also be of interest (Figure 3). As a result, our study suggests considering latitude- and altitude-dependence gravity accelerations as well as a latitude-dependence radius within the computation of the inner integral. 


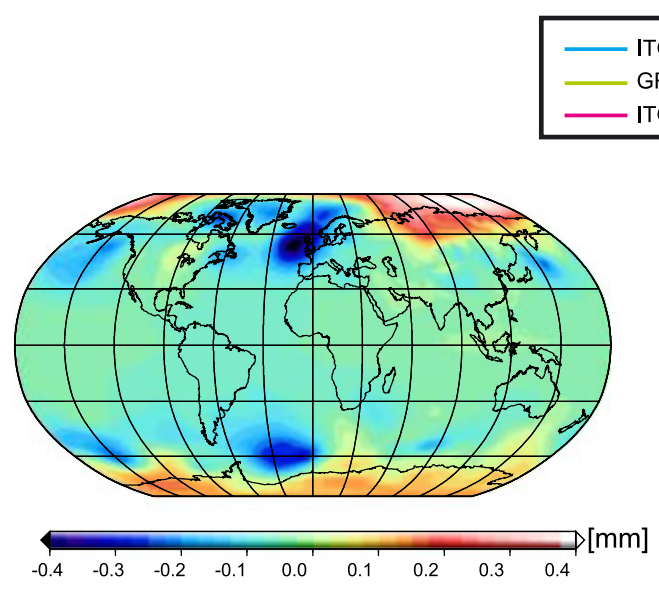

(a)

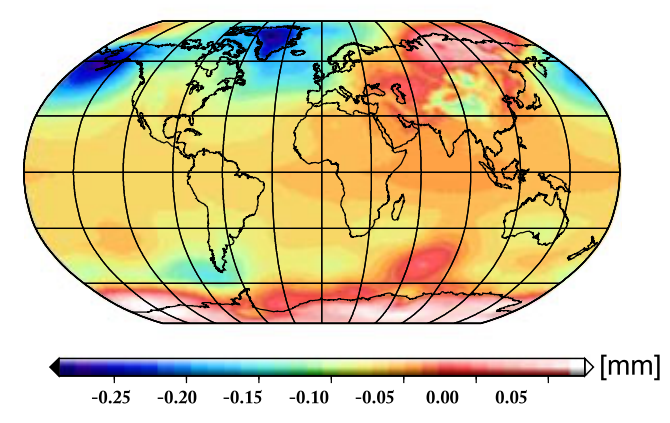

(c)

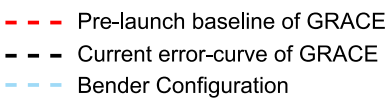

- - - Pre-launch baseline of GRACE

- - - Current error-curve of GRACE Bender Configuration

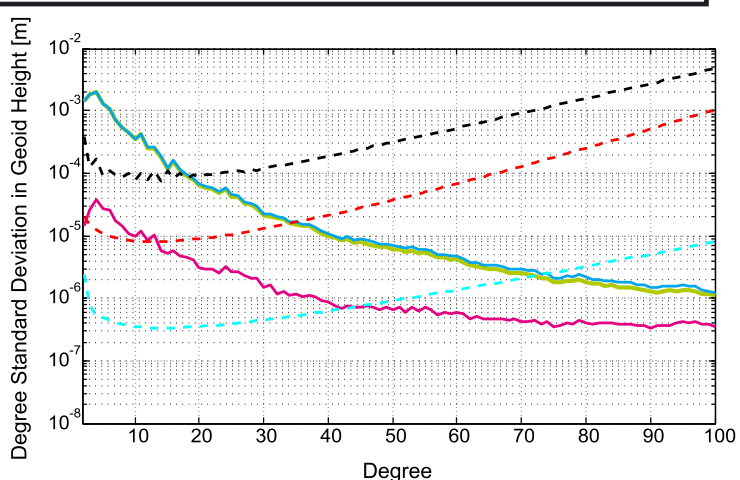

(b)

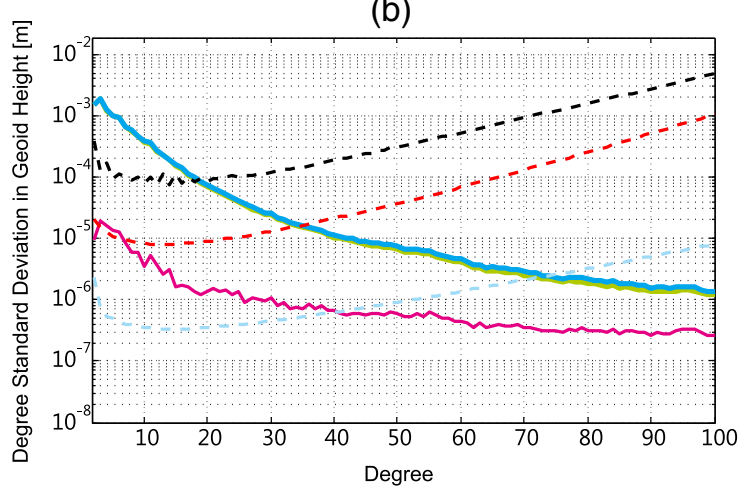

(d)

Figure 5. Comparison between daily and monthly anomalies derived from ITG-3-D and GRACEAOD1B. (a) spatial pattern of daily differences, based on the ECMWFop data on 1 January 2001, (b) daily anomalies of Figure 5a, in terms of degree standard deviations of geoid height, (c) spatial pattern of monthly averaged of differences between ITG-3-D and GRACE-AOD1B, on January 2001, and (d) monthly anomalies of Figure 5c, in terms of degree standard deviations. Dotted lines show the sensitivity of gravity missions. Solid blue and solid green lines denote the dealiasing products computed using ITG-3-D and the atmospheric part of AOD1B, respectively. Solid magenta line in Figure $5 \mathrm{~b}$ denotes the daily difference which is above the prelaunch baseline of GRACE and the instrumental error-curve of a Bender-type mission up to degrees 13 and 43, respectively. Solid magenta line in Figure 5d denotes the monthly difference, whose amplitude is reduced (compared to the daily difference) due to averaging. Monthly difference is found above the prelaunch baseline of GRACE and the instrumental error-curve of a Bender mission respectively up to degrees 7 and 40 .

\subsection{Comparisons Between ITG-3-D and GRACE-AOD1B Products}

[42] In the following, we compare the suggested ITG-3-D approach for computing atmospheric dealiasing products to that of GRACE-AOD1B in three ways: (1) Computing differences for six-hourly dealiasing products, to compare the impact of the two approaches for revealing short wavelength atmospheric variability. This impact is relevant since dealiasing products would be directly reduced from the level 1 measurement, while high-frequency (sixhourly) mass redistributions are undersampled by satellite passes. (2) Computing daily averaged differences, to compare their impacts with respect to short-period atmospheric mass redistributions. (3) Computing averaged differences over 30 day intervals, in order to approximate the average effect of the products on monthly solutions. The monthly impact is of interest since, for computing gravity solutions from the observations of satellite gravimetry missions, it is common to average measurements collected over, e.g., 30 day periods. It should be mentioned here that, in the process of computing GRACE-AOD1B products, the mean term $\left(\bar{I}_{n}\right)$ for the years 2001 and 2002 is reduced within the computation of the dealiasing products [Flechtner, 2007; Flechtner et al., 2010]. This reduction itself has an impact on the computation of spherical harmonics in equation (1). In section 4.4, we will discuss this impact on the computation of the new dealiasing products. To reduce the effect of the mean field reduction on the comparisons that are shown in this paper, the term $\bar{I}_{n}$ has been separately computed with respect to the chosen method from Table 1 and is removed within computing equation (2). 


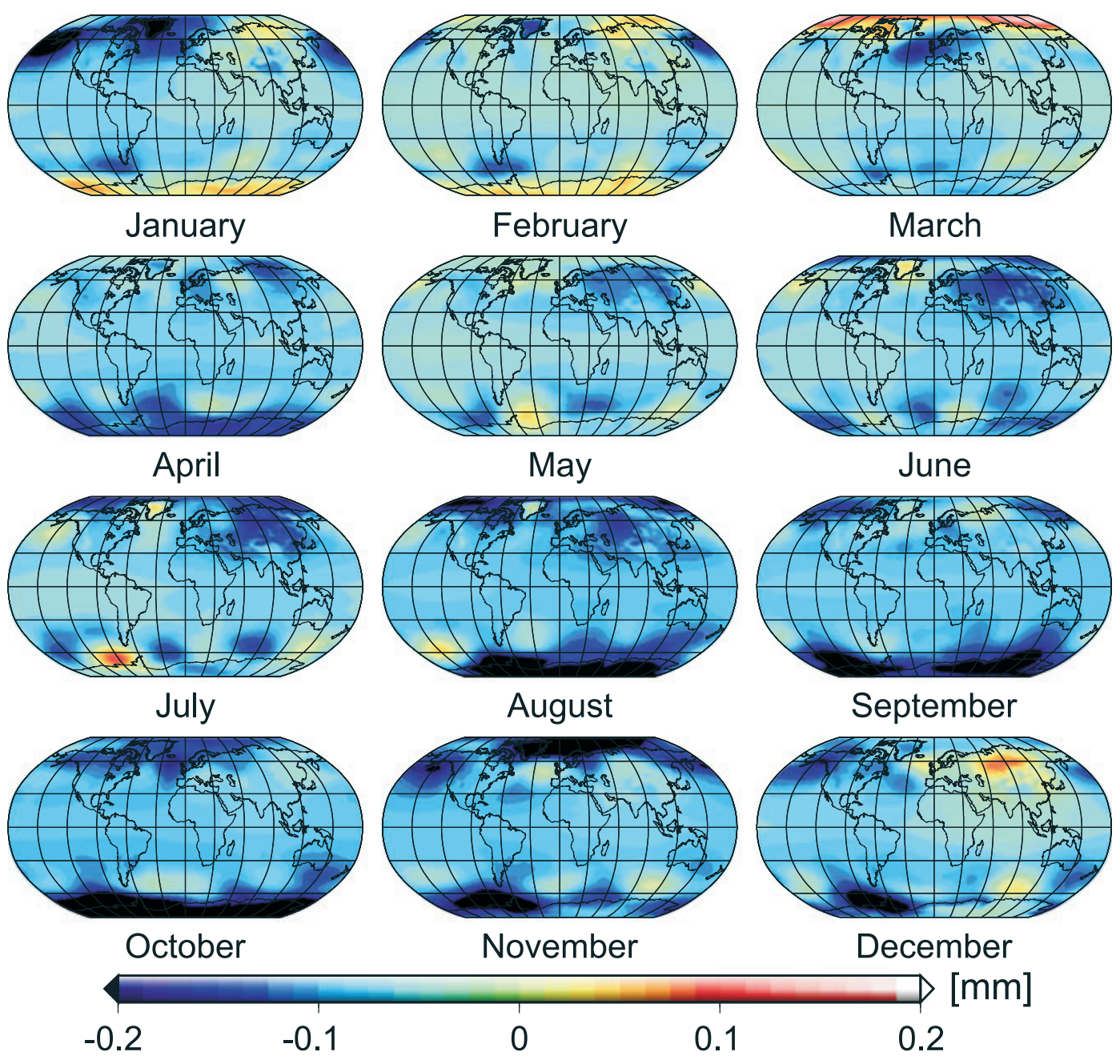

Figure 6. Monthly mean differences between ITG-3-D and GRACE-AOD1B, in terms of geoid height, for the year 2001. The products are computed based on the ECMWFop data.

[43] Figure 4 shows the difference between atmospheric loading computed from ITG-3-D [inserting equation (15), with considering the discussed numerical improvements from section 3.4, in equation (1)] and GRACE-AOD1B [inserting equation (13), without further numerical modifications, in equation (1)]. The computation used the ECMWFop data on 1 January 2001 at 00:00 h, while $\bar{I}_{n}$ was derived from the mean field of the years 2001 and 2002 [respectively, from the temporal average of equations (15) and (13) without considering $\left.\bar{I}_{n}\right]$. The result shows a nonnegligible difference between the suggested method and GRACE-AOD1B up to degrees 13 and 50 respectively with compared to the prelaunch baseline of GRACE and the instrumental errorcurve of a Bender mission. The differences in Figure $4 \mathrm{~b}$ are presented in terms of degree standard deviations of geoid height.

[44] For computing daily anomalies, from both ITG-3-D and GRACE-AOD1B, four products of each day were temporally averaged. Figures $5 \mathrm{a}$ and $5 \mathrm{~b}$ compare the differences between the daily anomalies on 1 January 2011. The spatial pattern of the daily difference shows a range of -0.4 to $0.4 \mathrm{~mm}$ (Figure $5 \mathrm{a}$ ). Figure $5 \mathrm{~b}$ shows the impacts of the daily anomaly, in terms of degree standard deviations of geoid height, where the difference is found above the prelaunch baseline of GRACE and the instrumental error-curve of a Bender-type mission up to degrees 13 and 43, respectively (Figure 5b).

[45] Monthly anomalies consist of temporal averages of the six-hourly dealiasing products over arbitrary months. Figures $5 \mathrm{c}$ and $5 \mathrm{~d}$ show the difference of monthly anomalies computed from ITG-3-D and GRACE-AOD1B on January 2001. The spatial pattern of differences is illustrated in Figure $5 \mathrm{c}$, showing a range of -0.25 to $0.05 \mathrm{~mm}$, in terms of geoid height. Monthly anomalies and their differences, in terms of degree standard deviations of geoid height, are summarized in Figure 5d. The results show that the monthly difference is above the prelaunch baseline of GRACE and the instrumental error-curve of a Bender mission up to degrees 7 and 40, respectively (Figure 5d).

[46] Comparing the patterns in Figures 5a and 5b respectively to those of Figures $5 \mathrm{c}$ and $5 \mathrm{~d}$, one can see that due to the averaging of the monthly anomaly, the amplitude of differences are reduced. Yet, the derived monthly differences are still considerable for computing satellite gravimetry 


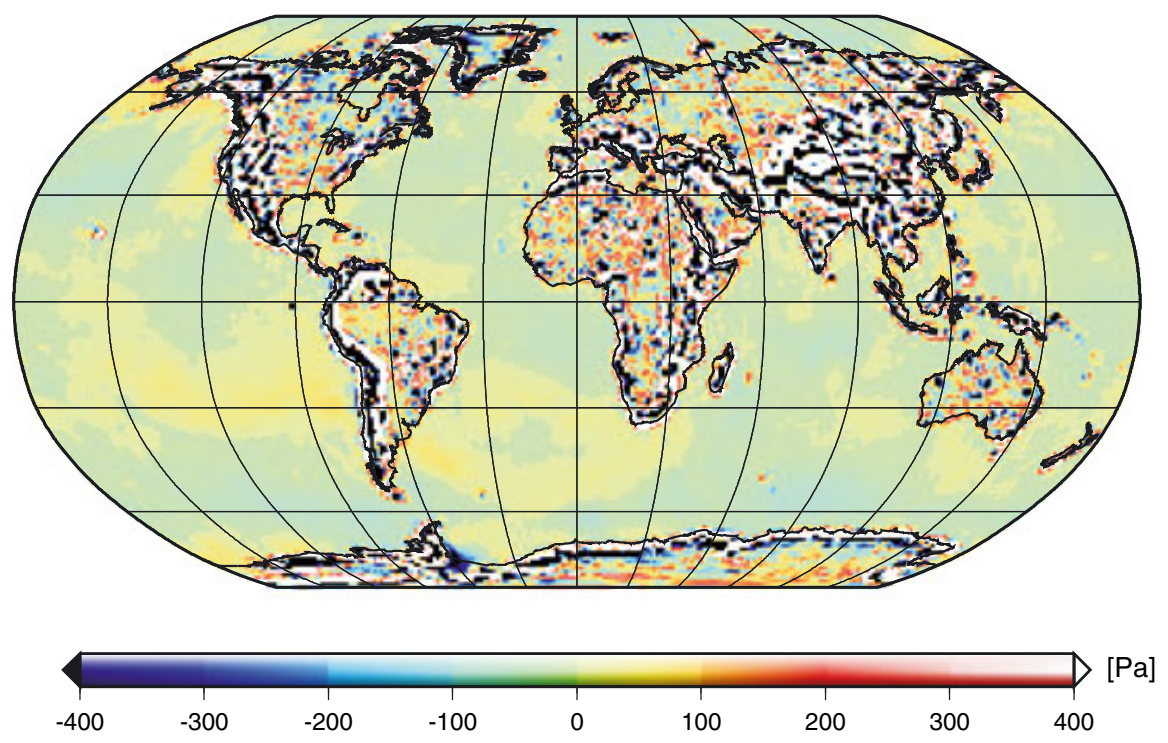

Figure 7. Monthly average of pressure differences (in Pascal) from ECMWFop and ERA-Interim on January 2001.

products with millimeter precision, in terms of geoid height [see NG2 Team, 2011]. Note also that, even for the current GRACE mission, the required accuracy of monthly cumulative error is less than $\sim 0.42 \mathrm{~mm}$, in geoid height, for a solution up to degree and order 70 (http://op. gfz-potsdam.de/grace/gravity/gravity.html). Therefore, we believe that, even though the derived differences between ITG-3-D and GRACE-AOD1B are in the range of submillimeters, they should be considered within the reprocessing of the current GRACE gravity field solutions.

[47] In order to illustrate the impact of using the different approaches to compute monthly gravity fields, we computed monthly mean differences between ITG-3-D and GRACE-AOD1B, similar to Figure 5c, for the whole year 2001. The results, in terms of geoid height, are shown in Figure6, indicating large differences over high latitude regions specifically toward the polar regions.

\subsection{Atmospheric Dealiasing Products Based on ERA-Interim}

[48] Comparing atmospheric fields from ERA-Interim with ECMWFop shows that there is a significant difference in their surface pressure fields $\left(P_{s}\right)$. For instance, the averaged surface pressure differences for January 2001 is shown in Figure 7, in which a maximum difference of 3 to $4 \mathrm{hPa}$ was detected. Salstein et al. [2008] also reported a similar magnitude of difference between the pressure fields of NCEP and ECMWFop.

[49] In order to illustrate the impact of the input atmospheric parameters on the computation of the atmospheric dealiasing products, we used data sets from ERA-Interim reanalysis. Then, a new set of atmospheric dealiasing products, based on our ITG-3-D approach and ERA-Interim data, was computed. Monthly averaged differences between the dealiasing products derived from ERA-Interim and those of ECMWFop, for the year 2001, are shown in Figure 8.

[50] The spatial patterns of Figure 8 show that the differences are mostly distributed over Antarctica and the southern oceans (see, e.g., the patterns of January, February, April, and July in Figure 8) and also over steep orography (e.g., Central Asia in July and August). The magnitude of the differences reaches up to $1 \mathrm{~mm}$, in terms of geoid height, which is only caused by using of ERA-Interim instead of the operational fields for the computation of dealiasing products. There might also be some seasonal differences between the dealiasing products computed from ERA-Interim and those of the operational model (Figure 8), which have impacts on the final gravity field products. Addressing such differences and their impacts needs further research.

\subsection{Impact of Atmospheric Dealiasing on a Monthly GRACE Solution}

[51] In the following, we compare the impact of different atmospheric dealiasing products on a GRACE monthly solution. For this computation, we used GRACE level-1B data of January 2009. To remove high-frequency hydrologic and oceanic mass changes from the level-1B data, the daily Kalman-filter ITG-GRACE solutions as well as the oceanic part of the GRACE-AOD1B RL04 were used, respectively. For reducing high-frequency atmospheric changes, however, we used different dealiasing products including the following: (1) the atmospheric part of GRACE-AOD1B (AOD1B/ECMWFop/mean2001+2002), (2) our ITG-3-D products based on ECMWFop, while a mean atmospheric field of 2001 and 2002 was reduced during the computation procedure (ITG-3D/ECMWFop/mean2001+2002), (3) our ITG-3-D products computed based on ECMWFop, while a mean atmospheric field of January 2009 was reduced during the computations (ITG-3D/ECMWFop/meanJan2009), and (4) the same product as (2) but based on the ERAInterim data (ITG-3D/ERA/mean2001+2002). (5) the same product as (3) but based on the ERA-Interim data (ITG3D/ERA/meanJan2009). Therefore, using the five mentioned atmospheric dealiasing products, five gravity field solutions on January 2009, up to degree and order 90, were computed following Mayer-Gürr [2006]. The differences between the computed solutions, in terms of geoid height, are summarized in Figure 9. 

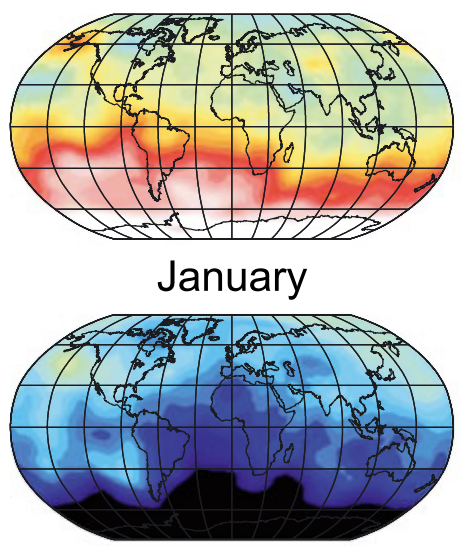

April

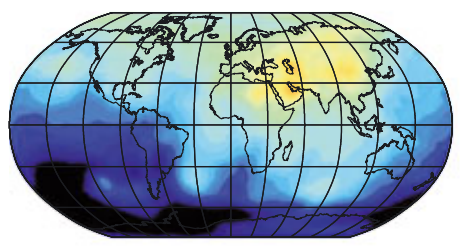

July

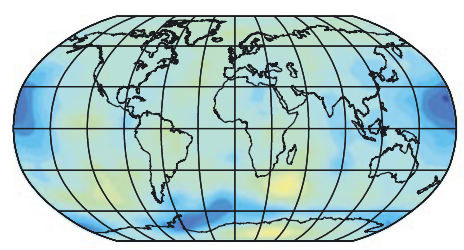

October

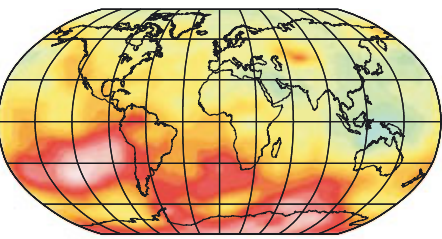

February

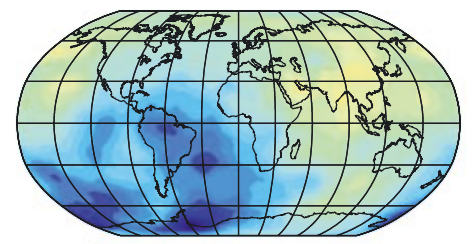

May

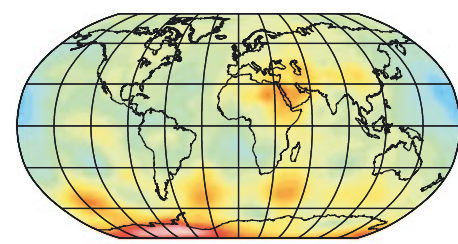

August

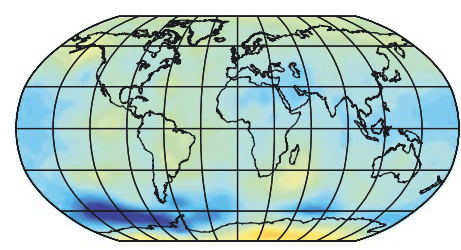

November

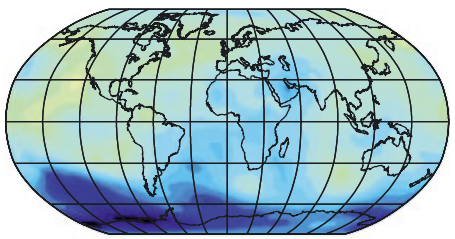

March

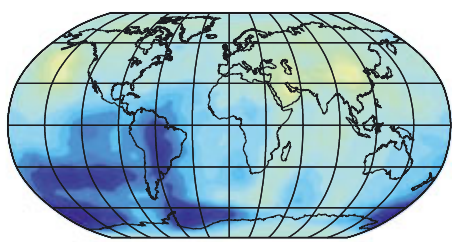

June

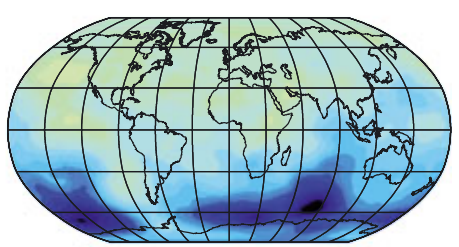

September

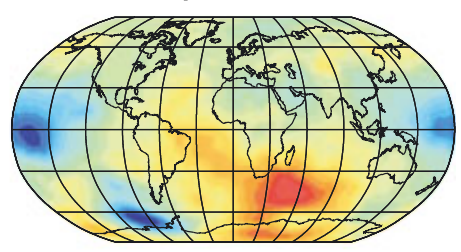

December

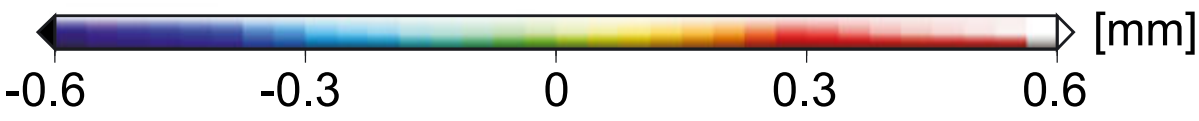

Figure 8. Monthly averaged differences between the dealiasing products derived from ERA-Interim and those from the ECMWFop, for the year 2001, in terms of geoid heights.

[52] The difference between two solutions that are reduced by product ii and the GRACE-AOD1B was found above the prelaunch baseline of GRACE up to degree 6, indicating its usefulness for the processing of GRACE-FO and future missions. In fact, the error-curve of the new computed GRACE-RL05 [Dahle et al., 2012] monthly solutions is much closer to the prelaunch baseline of GRACE than the current error-curve of GRACE we showed here. Therefore, the ITG-3-D products might also be beneficial for reprocessing of the mission GRACE data.

[53] The difference between using products ii and iv in the solution of January 2009 was found above the prelaunch baseline of GRACE, up to degree 67. This impact indicates the importance of input atmospheric fields (i.e., ERA-Interim instead of ECMWFop) on a GRACE gravity field solution. However, neither the impact of the modeling modification nor the reanalysis data are above the current accuracy of GRACE monthly gravity field solutions.

[54] As mentioned before, the atmospheric mean field removed during the computation of the atmospheric dealiasing products itself is a factor that affects the dealiasing products. To assess such effect on the GRACE solution, the difference of two solutions, computed by reducing products ii and iii, as well as the difference between two solutions based on products iv and $\mathrm{v}$. The results show that the impact of the mean field is above the current error-curve of GRACE up to degree 11 (Figure 9). The reason for this difference is that high-frequency mass variations are better represented in those atmospheric products in which the monthly $\bar{I}_{n}$ are removed during the computation procedure, rather than the 2 year $\bar{I}_{n}$. As a result, those GRACE mean fields that are computed while removing the mean of the corresponding month indicate less RMS than those based on 2 year mean reduction. This might be concluded as improvement with respect to error reduction. From a hydrological perspective, however, one should keep in mind that incorrectly removed long wavelength atmospheric mass variabilities (e.g., atmospheric mass changes with the period of 1 month to several years) also have an adverse effect on the interpretation of gravity signals presented in the final gravity field solutions. Therefore, including those differences in the process of computing atmospheric dealiasing products is still an issue which needs further research. 


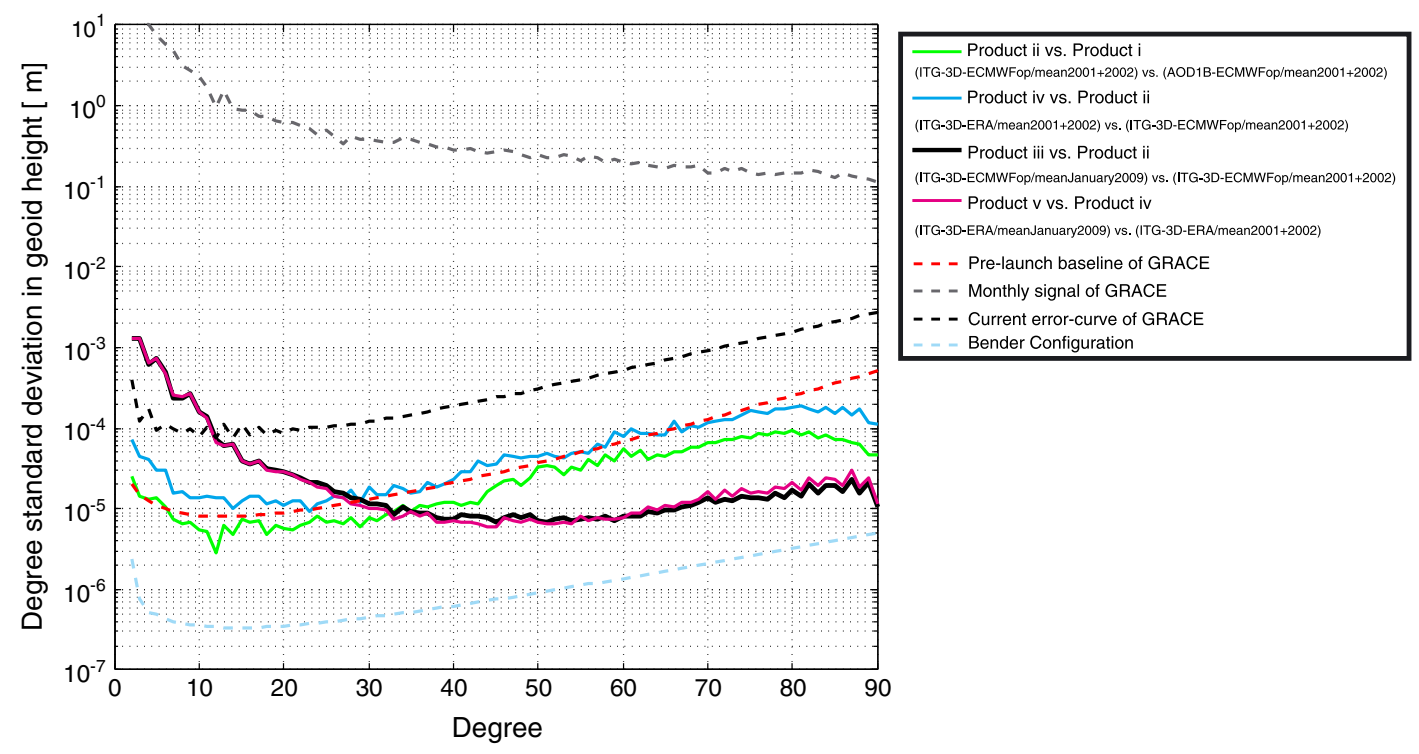

Figure 9. Impact of using different atmospheric dealiasing products on a GRACE monthly solution, on January 2009, computed up to degree and order 90, in terms of geoid height. In this figure, dotted lines show the sensitivity of gravity missions, and solid lines compare the impacts of different atmospheric dealiasing products (see section 4.4). Considering product $\mathrm{i}$ : the atmospheric part of GRACE-AOD1B (AOD1B/ECMWFop/mean2001+2002); product ii: ITG-3-D based on ECMWFop while a mean atmospheric field of 2001 and 2002 was reduced during the computation (ITG-3D/ECMWFop/mean2001+2002); product iii: our ITG-3-D based on ECMWFop while a mean atmospheric field of January 2009 was reduced (ITG-3-D/ECMWFop/meanJanuary2009); product iv: the same product as ii but based on ERA-Interim (ITG-3-D/ERA/mean2001+2002); and product v: the same product as iii but based on the ERA-Interim data (ITG-3-D/ERA/meanJanuary2009). Solid blue line denotes the difference between using product ii and product iv which shows the impact of the input atmospheric fields (i.e., ERA-Interim instead of ECMWFop). Solid green line denotes the difference between two solutions that are reduced by our ITG-3-D method (product ii) and the GRACE-AOD1B (product i), showing the impact of the modifications. Solid black and solid magenta lines denote the difference between two solutions that are computed by reducing products ii and iii and products iv and $\mathrm{v}$. Both lines show the impact of mean reduction.

\section{Discussions and Conclusions}

[55] Within this study, previous 3-D modeling approaches for calculating high-frequency atmospheric gravitational effects from operational atmospheric models were revisited. Impacts of different geometric, physical, and numerical approximations within the 3-D integration method were discussed. The results were compared with the prelaunch baselines of GRACE and its actual error-curve as well as the instrumental error-curve of a possible Bender-type configuration of future gravity field missions. Based on our results, the method called ITG-3-D, which takes the structure of the atmosphere and the shape of the Earth in a more realistic way into account, shows some significant differences compared to the previous methods of computing atmospheric dealiasing products. Considering the notable noise reduction and signal improvement of GRACE-RL05 products compared to its precursor RL04, the error-curve of the GRACE mission is now much closer to its prelaunch baseline. Therefore, considering the ITG-3-D method for reducing the atmospheric aliasing effect might be desirable.

[56] Our results from ERA-Interim also showed that computing dealiasing products with respect to a reanalysis model (i.e., ERA-Interim) can considerably change the final dealiasing products and consequently change the desired gravity field solutions. This result suggests that apart from an improved computation approach, impacts of the input parameters themselves represent an issue which needs more investigations. Due to more rigorous numerical procedures, we believe that the new method (ITG-3-D) represents a step toward computing more accurate dealiasing products, although only extended validation experiments will be able to prove this claim.

[57] Acknowledgments. The authors thank three anonymous reviewers for their helpful remarks which improved considerably the manuscript. We also thank F. Flechtner (GFZ, Potsdam) and C. Ohlwein (Meteorological Institute, Bonn University) for their help and comments during the computation stage of this study. The authors further wish to thank E. Kurtenbach (Institute of Geodesy and Geoinformation, Bonn University) for computing GRACE monthly solutions and J. M. Brockmann (Institute of Geodesy and Geoinformation, Bonn University) for his computational supports. J. Kusche is grateful for the financial support by the German Research Foundation (DFG), SPP1257: Mass transport and mass distribution in the Earth system. E. Forootan is grateful for the financial supports by the DFG under the project BAYES-G. The authors thank Deutscher Wetterdienst, Offenbach, Germany, and the European Centre for MediumRange Weather Forecasts, Reading, UK, for providing data from ECMWF's operational forecast model to the German Research Centre for Geosciences (GFZ) Potsdam, who made it available via its ISDC archive to users of DFG SPP1257 projects. We are also grateful to the ECMWF ERA-Interim data downloaded from http://data-portal.ecmwf.int/data/d/interim_daily/. 


\section{References}

Anselmi, A. (2010), Assessment of a next generation mission for monitoring the variations of Earth's gravity, Final Rep., Thales Alenia Space, SD-RP-AI-0688.

Bender, P. L., J. L. Hall, J. Ye, and W. M. Klipstein (2003), Satellite-satellite laser links for future gravity missions, Space Sci. Rev., 108, 377-384, doi: 10.1023/A:1026195913558.

Berrisford, P., D. Dee, K. Fielding, M. Fuentes, P. Kallberg, S. Kobayashi, and S. Uppala, (2009), The ERA-interim archive, Tech. Rep., The ERA-Interim archive - Version 1.0, ERA reports. http://www.ecmwf.int/ publications/, accessed date: January 2012.

Boy, J.-P., and B. F. Chao (2005), Precise evaluation of atmospheric loading effects on Earth's time-variable gravity field, J. Geophys. Res., 110, 1-10, doi:10.1029/2002JB002333.

Colombo, O. L., (1981), Numerical methods for harmonic analysis on the sphere, Number 310 in Reports of the Department of Geodetic Science, Ohio State University (OSU), Columbus, Ohio.

Dahle, Ch., F. Flechtner, Ch. Gruber, D. König, R. König, G. Michalak, and K. H. Neumayer, (2012), GFZ GRACE Level-2 processing standards document for Level-2 product release 0005, Scientific Technical Report STR12/02-Data, GFZ, Potsdam, Germany.

Dee, D. P., et al. (2011), The ERA-interim reanalysis: Configuration and performance of the data assimilation system, Q. J. R. Meteorol. Soc., 137, 553-597, doi:10.1002/qj.828.

Dehne, M., F. Guzmán-Cervantes, B. Sheard, G. Heinzel, and K. Danzman (2009), Laser interferometer for spaceborn mapping of the Earth's gravity, J. Phys. Conf. Ser., 154, 1-6, doi:10.1088/1742-6596/154/1/012023.

Dong, D., R. S. Gross, and J. O. Dickey (1996), Seasonal variations of the Earth's gravitational field: An analysis of atmospheric pressure, ocean tidal, and surface water excitation, Geophys. Res. Lett., 23(7), 725-728.

Eicker, A. (2008), Gravity field refinement by radial basis functions from in-situ satellite data, $\mathrm{PhD}$ dissertation, Bonn University, Germany.

Elsaka, B. (2010), Simulated satellite formation flights for detecting the temporal variations of the Earth's gravity field, $\mathrm{PhD}$ dissertation, Bonn University, Germany.

Elsaka, B., J. Kusche, and K.-H. Ilk (2012), Recovery of the Earth's gravity field from formation-flying satellites: Temporal aliasing issues, Adv. Space Res., 50(11), 1534-1552, doi:10.1016/j.asr.2012.07.016.

Fagiolini, E., L. Zenner, F. Flechtner, Th. Gruber, G. Schwarz, T. Trautmann, and J. Wickert (2007), The sensitivity of satellite gravity field determination to uncertainties in atmospheric models, joint GSTM/SPP Kolloquium, Potsdam, http://www.massentransporte. de/fileadmin/20071015-17-Potsdam/di 180002 fiagolini.pdf, accessed date: January 2012.

Farrel, W. E. (1972), Deformation of the Earth by surface loads, Rev. Geophys., 10, 761-797.

Flechtner, F. (2007), AOD1B product description document for product releases 01 to 04 , Tech. Rep., Geoforschungszentrum, Potsdam.

Flechtner, F., M. Thomas, and H. Dobslaw (2010), Improved non-tidal atmospheric and oceanic de-aliasing for GRACE and SLR satellites, Adv. Technol. Earth Sci., Part 2, 2010, 131-142, doi:10.1007/978-3-64210228-8 11

Gill, A. E. (1999), Atmosphere-Ocean Dynamics, 662 pp., Cambridge University Press, New York, ISBN:978-0122835223.

Gruber, Th., Th. Peters, and L. Zenner (2009), The role of the atmosphere for satellite gravity field missions, in Observing Our Changing Earth, IAG Symposia, vol. 133, edited by M. G. Sideris, pp. 105-112, Springer Berlin Heidelberg, doi:10.1007/978-3-540-85426-5.

Haltiner, G. J., and R. T. Williams (1979), Numerical Prediction and Dynamic Meteorology, 2nd ed., 477 pp., John Wiley \& Sons, New York.

Han, S., C. Jekeli, and C. Shum (2004), Time-variable aliasing effects of ocean tides, atmosphere, and continental water mass on monthly mean GRACE gravity field, J. Geophys. Res., 109, B04403, doi 10.1029/2003JB002501.

Heiskanen, W. A., and H. Moritz (1967), Physical Geodesy, 364 pp., W. H. Freeman \& Co Ltd, San Francisco, Calif., and London, U.K.

Karbon, M., D. Wijaya, M. Schindelegger, J. Böhm, and H. Schuh (2011), Atmospheric effects on the Earth gravity field featured by TU Vienna, Österreichische Zeitschrift für Vermessung und Geoinformation, 99. Jahrgang, Heft 2/2011, 122-130.

Kim, J. R. (2000), Simulation study of a low-low satellite-to-satellite tracking mission, $\mathrm{PhD}$ dissertation, University of Texas, Austin, Tex.

Krylov, V. I. (1962), Approximate Calculation of Integrals, 357 pp., MacMillan, New York. ISBN:978-0486445793.

Kurtenbach, E., T. Mayer-Gürr, and A. Eicker (2009), Deriving daily snapshots of the Earth's gravity field from GRACE L1B data using Kalman filtering, Geophys. Res. Lett., 36(7), L17102, doi:10.1029/ 2009GL039564.
Kusche, J., V. Klemann, and W. Bosch (2012), Mass distribution and mass transport in the Earth system, J. Geodyn., 59-60, 1-8, doi:http://dx.doi. org/10.1016/j.jog.2012.03.003.

Loomis, B. D., R. S. Nerem, and S. B. Luthcke (2012), Simulation study of a follow-on gravity mission to GRACE, J. Geod., 86, 319-335, doi: 10.1007/s00190-011-0521-8.

Lorenz, C., and H. Kunstmann (2012), The hydrological cycle in three state-of-the-art reanalyses: Intercomparison and performance analysis, J. Hydrometeorol, 13, 1397-1420, doi:10.1175/JHM-D-11-088.1.

Mayer-Gürr, T. (2006), Gravitationsfeldbestimmung aus der Analyse kurzer Bahnbögen am Beispiel der Satellitenmissionen CHAMP und GRACE PhD dissertation, Bonn University, Germany.

Mayer-Gürr, T., A. Eicker, E. Kurtenbach, and K. H. Ilk (2010a), ITG-GRACE: Global static and temporal gravity field models from GRACE data, Advanced Technologies in Earth Sciences, 159-168, doi: 10.1007/978-3-642-10228-8_13

Mayer-Gürr, T., E. Kurtenbach, and A. Eicker (2010b), Different representations of the time variable gravity field to reduce the aliasing problem in GRACE data analysis, Proceedings VII Hotine-Marussi Symposium on Theoretical and Computational Geodesy, 2010.

Mayer-Gürr, T., E. Kurtenbach, and A. Eicker $(2010 \mathrm{c})$, ITGGrace2010. Available at http://www.igg.uni-bonn.de/apmg/index.php? id=itg-grace2010, accessed March 2013 .

Meyer, U., F. Flechtner, R. Schmidt, and B. Frommknecht (2010), A simulation study discussing the GRACE baseline accuracy, in Gravity, Geoid and Earth Observation. IAG Commission 2: Gravity Field, Chania, Crete, Greece, 23.-27.6.2008. International Association of Geodesy Symposia, vol. 135, edited by S. P. Mertikas, pp. 171-176, Springer, Berlin, Heidelberg, doi:10.1007/978-3-642-10634-7 23.

Nerem, R. S., P. Bender, B. Loomis, M. M. Watkins, W. M. Folkner, M. Stephens, R. Craig, J. Leitch, and R. Pierce (2006), Development of an interferometric laser ranging system for a follow-on gravity mission to GRACE, Geophysical Research Abstracts, Vol. 8, 08685, SRef-ID 1607-7962/gra/EGU06-A-08685, European Geosciences Union, Vienna, Austria.

NG2 Team (2011), Assessment of a next generation gravity mission to monitor the variations of Earth's gravity field, Final Rep., European Space Agency (ESA) and Astrium GmbH.

Office of the Federal Coordinator for Meteorological Services and Supporting Research (OFCM) (1997), Federal Meteorological Handbook No. 3: Rawinsonde and Pibal Observations, FCM-H3-1997, Silver Spring, Md. (available online at http://www.ofcm.gov/fmh3/text/, accessed date: March 2012).

Peters, T. (2007), Modellierung zeitlicher Schwerefeldvariationen und ihre Erfassung mit Methoden der Satellitengravimetrie, $\mathrm{PhD}$ dissertation, Technical University of Munich.

Pierce, R., J. Leitch, M. Stephens, P. Bender, and R. S. Nerem (2008), Intersatellite range monitoring using optical interferometry, Appl. Opt., 47, 5007-5018, doi:10.1364/AO.47.005007.

Reigber, C., R. Schmidt, F. Flechtner, R. König, U. Meyer, K. H. Neumayer, P. Schwintzer, and S. Y. Zhu (2005), An Earth gravity field model complete to degree and order 150 from GRACE: EIGENGRACE02S, J. Geodyn., 39, 1-10, doi:10.1016/j.jog.2004.07.001.

Salstein, D. A., R. M. Ponte, and K. Cady-Pereira (2008), Uncertainties in atmospheric surface pressure fields from global analyses, J. Geophys. Res., 113, D14107, doi:10.1029/2007JD009531.

Schrama, E. J. O., B. Wouters, and D. D. Lavallee (2007), Signal and noise in Gravity Recovery and Climate Experiment (GRACE) observed surface mass observations, J. Geophys. Res., 112(B08407), doi: $10.1029 / 2006$ JB004882.

Sheard, B. S., G. Heinzel, K. Danzmann, D. A. Shaddock, W. M. Klipstein, and W. M. Folkner (2012), Intersatellite laser ranging instrument for the GRACE follow-on mission, J. Geod., 86(12), 1083-1095, doi:10.1007/s00190-012-0566-3.

Simmons, A. J., and D. M. Burridge (1981), An energy and angular momentum conserving vertical finite difference scheme and hybrid vertical coordinates, Mon. Wea. Rev., 109, 758-766, doi: http://dx.doi.org/10.1175/1520-0493(1981)109<0758:AEAAMC >2. $0 . \mathrm{CO} ; 2$.

Sneeuw, N. (1994), Global spherical harmonic analysis by least squares and numerical quadrature methods in historical perspective, Geophys. J. Int. 118, 707-716, doi:10.1111/j.1365-246X.1994.tb03995.X.

Sneeuw, N., J. Flury, and R. Rummel (2004), Science requirements on future missions and simulated mission scenarios, Earth, Moon, and Planets, 94, 113-142, doi:10.1007/s11038-004-7605-X.

Stockdale, T. N., D. L. T. Anderson, J. O. S. Alves, and M. A. Balmaseda (1998), Global seasonal rainfall forecasts using a coupled ocean-atmosphere model, Nature, 392, 370-373, doi:10.1038/ 32861 . 
Swenson, S., and J. Wahr (2002), Estimated effects of the vertical structure of atmospheric mass on the time-variable geoid, J. Geophys. Res., 107(9), 2194, doi:10.1029/2000JB000024.

Tapley, B. D., S. Bettadpur, J. C. Ries, P. F. Thompson, and M. M. Watkins (2004), GRACE measurements of mass variability in the Earth system, Science, 305, 503-505, doi:10.1126/science.1099192.

Thompson, P. F., S. V. Bettadpur, and B. D. Tapley (2004), Impact of short period, non-tidal, temporal mass variability on GRACE gravity estimates, Geophys. Res. Lett., 31, L06619, doi:10.1029/ 2003 GL019285.

Velicogna, I., J. Wahr, and H. Van den Dool (2001), Can surface pressure be used to remove atmospheric contributions from GRACE data with sufficient accuracy to recover hydrological signals?, J. Geophys. Res., 106(B8), 16415-16434, doi:10.1029/2001JB000228.

Visser, P. N. A. M., N. Sneeuw, T. Reubelt, M. Losch, and T. van Dam (2010), Space-borne gravimetric satellite constellations and ocean tides: Aliasing effects, Geophys. J. Int., 181, 789-805, doi: 10.1111/j.1365246X.2010.04557.X.

Wahr, J., M. Molenaar, and F. Bryan (1998), Time variability of the Earth's gravity field: Hydrological and oceanic effects and their possible detection using GRACE, J. Geophys. Res., 103(B12), 30205-30229, doi:10.1029/98JB02844.
Wang, H., P. Wu, and Z. Wang (2006), An approach for spherical harmonic analysis of non-smooth data, Comput. Geotech., 32, 1654-1668, doi:10.1016/j.cageo.2006.03.004

White, P. W. (2001), IFS documentation part III: Dynamics and numerical procedures (CY21R4), ECMWF Research Department, http:// www.ecmwf.int/research/ifsdocs/CY33r1/DYNAMICS/IFSPart3.pdf, accessed date: March 2012

Wiese, D. N., W. M. Folkner, and R. S. Nerem (2009), Alternative mission architectures for a gravity recovery satellite mission, J. Geod., 83, 569-581, doi:10.1007/s00190-008-0274-1.

World Meteorological Organization (WMO) (2006), WMO guide to meteorological instruments and methods of observation, WMO-No.8 Rep., Secretariat of the World Meteorological Organization, Geneva, Switzerland.

Zenner, L., T. Gruber, A. Jäggi, and G. Beutler (2010), Propagation of atmospheric model errors to gravity potential harmonics-Impact on GRACE de-aliasing, Geophys. J. Int., 182, 797-807, doi:10.1111/j.1365246X.2010.04669.X

Zenner, L., E. Fagiolini, I. Daras, F. Flechtner, T. Gruber, T. Schmidt, and G. Schwarz (2012), Non-tidal atmospheric and oceanic mass variations and their impact on GRACE data analysis, J. Geodyn., 59-60, 9-15, doi: http://dx.doi.org/10.1016/j.jog.2012.01.010. 